\title{
Tracking and Regulation Control of a 2-DOF Robot Arm with Unbalance
}

\author{
Samet Güler, A. Bülent Özgüler \\ Department of Electrical and Electronics Engineering \\ Bilkent University, Turkey \\ guler@ee.bilkent.edu.tr, ozguler@ee.bilkent.edu.tr
}

\begin{abstract}
Servomechanism synthesis for a two degree-offreedom robot arm assembled on a vessel and affected from sea disturbances is considered. Dynamic equations of the robot arm with unbalance and of an approximate base disturbance model are derived. Direct linear controller synthesis based on a linearized model is then studied. It is shown that, although the plant model is nonlinear, linear controller that is synthesized based on the "internal model principle" provide good performance and perform better than the commonly used PID controllers.
\end{abstract}

\section{INTRODUCTION}

Mathematical models of robots contain highly nonlinear terms because of the coupling between joints. In robots of more than one degree-of-freedom (DOF), nonlinear terms appear as coriolis and centrifugal forces. Such nonlinearities impede the design of controllers that meet desired specifications. While the use of robots on vessels are becoming popular, disturbances that come from sea through the vessel induce more nonlinear terms and controller design gets even more involved, [15]. In this work, a two degree-offreedom Revolute-Revolute (RR) joint robot arm mounted on a vessel and is influenced by sea disturbances is modeled. The disturbance modeling is the main issue and [15], [5], and [6] focus on this with the objective of compensating the effects of these disturbances on robots. On the other hand, in [11], the authors use a combination of a linear and nonlinear controller and an adaptive algorithm, where the linear agorithm is based on internal model principle to regulate disturbances, to solve the tracking and regulation problem for uncertain mechanical systems. Here, as in [11], the main emphasis will be on controller synthesis and whether a linear controller would be able to regulate (asymptotically quench or reject) disturbances while achieving the tracking and internal stability objectives. Furthermore, motor dynamics are neglected and nonlinear effects such as friction, backlash, etc., are assumed to be exactly compensated and are not considered since their compensation requires techniques like adaptive, robust, and neural control algorithms, [18]. Servomechanism syntheses that achieve tracking, sea disturbance regulation, and (internal) stability will thus be considered through this work.

One way to control a robot arm is to use Computed Torque Controllers, [13]. In this method, by using a suitable inverse of the nonlinearities in the model as part of an overall nonlinear controller, the system becomes a decoupled linear error dynamics system which comprises a double integrator for each link. A further linear controller can then be employed to achieve other objectives. In this method, the tracking objective is automatically achieved via internal stabilization of the error system, because the signal to be tracked is already incorporated as part of the nonlinear controller. As long as the nonlinear control implemented remains intact, this method provides good tracking ability. However, when it comes to elimination of external disturbances, then adaptive, robust or other complex structures need to be used along with this method. This is because the resulting error system does not allow the use of internal model principle directly for regulation. There have been attempts to reconcile the internal model principle and the computed torque method. For instance, in [14], the linear plant is pre-stabilized and a linear controller based on the internal model principle is then employed for regulation. An alternative to the computed torque control is the direct approach of linearizing the model and employing a linear controller. This second method may usually fail because linearization, in most cases, drops the coriolis and centrifugal terms, and produces an oversimplified approximate model, [2]. Linearization is hence hardly considered in the control of robot arms. We will try to establish however that the relative advantages of these two methods are not so clear as is usually thought.

The robot model obtained here can be thought of as the first two links of a PUMA 560 robot arm, [16], upon which an approximation of effects of disturbances caused by sea movements is also incorporated. The model obtained is standard and is similar to those one finds in the literature, [5], [6], [15], [16]. The obtained base disturbance model is then represented as an external disturbance and the resulting simplified model is then linearized about a natural operating point. Two different linear controllers are designed for this linearized plant and they are implemented on the nonlinear model. The first is a PID controller, the parameters of which are tuned for the best observed performance. Of course, such a controller will be able to track and regulate step signals but not sinusoidals even if the plant had been linear. It turns out that this expectation is also fulfilled for our nonlinear plant and PID controller tracks and regulates step signals and tracks but can not regulate ramp signals. The second is an appropriate linear controller that is synthesized by evoking the "internal model principle" [4]. Simulations show that the internal model based controller is able to regulate step or sinusoidal type disturbance signals and is able to track step or sinusoidal reference signals (commands) 
satisfactorily. Naturally, it is assumed for sinusoidal tracking and regulation that the frequency content of the reference and disturbance signals are known. One further observation that results by the linearization method is that, if the upper link has unbalance (mismatch between the joint location and the center of gravity), then it should be compensated before a linear controller is synthesized.

Internal model principle primarily applies to linear, timeinvariant controller synthesis for the purpose of driving the output of a system asymptotically to zero in a feedback loop and states that such regulation can only be achieved if the loop (or controller) contains an internal model of the input signal being regulated, [4]. The principle loses its generality when applied to nonlinear systems although, in [8], [9], [10], and in [1], [3] one finds instances of such applications. As will be shown below, the fact that internal model principle applies to the robot model considered here can be interpreted to mean that the 2-DOF, RR robot arm model obtained here is "close to being linear" with respect to the objectives of internal stability, regulation, and tracking.

The paper is organized as follows. In Section 2, the dynamic model of a 2-DOF robot arm is obtained and a disturbance model which represents the sea disturbances is incorporated. Controller synthesis and simulation via linearization and internal model principle after the control of unbalance is given in Section 3. The last section is on conclusions.

\section{DyNAMIC MODELING}

\section{A. Dynamic Model of 2-DOF RR Robot}

A rigid two degree-of-freedom revolute-revolute joint robot can be represented as in Fig. 1 and can be modeled by using Lagrange-Euler Formulation as described in [7] and [19]. Suppose the second link contains an unbalance, i.e., the center of mass of the second link is not on the axis of revolution of the first link (See [20] for more details).

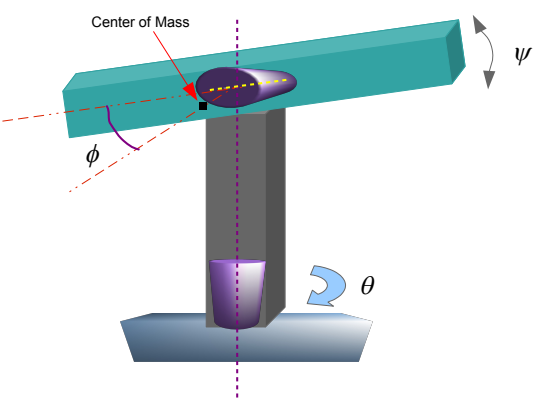

Fig. 1. 2 Degree-Of-Freedom RR Robot Arm

Dynamic equation of the robot arm is

$\left[\begin{array}{cc}M_{11} & 0 \\ 0 & M_{22}\end{array}\right]\left[\begin{array}{c}\ddot{\theta} \\ \ddot{\psi}\end{array}\right]+\left[\begin{array}{l}N_{1} \\ N_{2}\end{array}\right]+\left[\begin{array}{l}G_{1} \\ G_{2}\end{array}\right]+\left[\begin{array}{l}\tau_{f 1} \\ \tau_{f 2}\end{array}\right]=\left[\begin{array}{l}\tau_{1} \\ \tau_{2}\end{array}\right]$, where $M_{11}, M_{22}$ are so called inertia terms, $N_{1}, N_{2}$ are coriolis and centrifugal forces, and $G_{1}, G_{2}$ are gravity related terms for the first and second joints, respectively, given by

$$
\begin{aligned}
& M_{11}=\zeta+\gamma \cos ^{2}(\psi)-p \sin (2 \psi), M_{22}=\eta, \\
& N_{1}=\dot{\theta} \dot{\psi}(-\gamma \sin (2 \psi)-2 p \cos (2 \psi)), \\
& N_{2}=\frac{1}{2} \dot{\theta}^{2}(\gamma \sin (2 \psi)+2 p \cos (2 \psi)), \\
& G_{1}=0, G_{2}=-\kappa \cos (\psi+\phi),
\end{aligned}
$$

with $\zeta, \gamma, p, \eta$ being inertia related constants. Also in (1), $\tau_{\mathbf{f}}=$ $\left[\tau_{f 1}, \tau_{f 2}\right]^{T} \in \mathbb{R}^{2 \times 1}$ represents the friction torque and $\mathbf{u}=$ $\left[\tau_{1}, \tau_{2}\right]^{T} \in \mathbb{R}^{2 \times 1}$ is the external torque input. The constant $\kappa$ has interpretation as the magnitude of the unbalance torque and $\phi$ is the unbalance angle indicated in Fig. 1.

Using the state vector $\mathbf{x}=[\theta, \dot{\theta}, \psi, \dot{\psi}]^{T} \in \mathbb{R}^{4 \times 1}$, the input vector $\mathbf{u}$, and separating the linear term in $\mathbf{x}$, equation (1) can be written in state-space representation

$$
\frac{\mathbf{d}}{\mathbf{d t}} \mathbf{x}=\mathbf{A} \mathbf{x}+\mathbf{f}(\mathbf{x}, \mathbf{u}, t), \quad \mathbf{y}=[\theta, \psi]^{T},
$$

with

$$
\mathbf{A}=\left[\begin{array}{llll}
0 & 1 & 0 & 0 \\
0 & 0 & 0 & 0 \\
0 & 0 & 0 & 1 \\
0 & 0 & 0 & 0
\end{array}\right], \mathbf{f}(\mathbf{x}, \mathbf{u}, t)=\left[\begin{array}{c}
0, \\
\mathbf{f}_{\mathbf{1}}(\mathbf{x}, \mathbf{u}, t) \\
0 \\
\mathbf{f}_{\mathbf{2}}(\mathbf{x}, \mathbf{u}, t)
\end{array}\right]
$$

where $\mathbf{f}_{\mathbf{i}}=\frac{\tau_{i}-N_{i}-G_{i}-\tau_{f i}}{M_{i i}} ; i=1,2$.

When the inputs $\tau_{1}, \tau_{2}$ and the friction torque vector $\tau_{\mathbf{f}}$ are zero, the open-loop response of this robot oscillates because of the unbalance term exists at the second link.

\section{B. Base Disturbance Model}

Sea disturbances are caused by sea waves, currents, or winds. The most important of these is the effect of sea waves which influences the robot through the vehicle dynamics and occur in all three principal $x y z$ coordinates. In [15], these effects are taken into account resulting in a 6-DOF (3 revolute and 3 prismatic joints) robot. Here, for simplicity, we consider a 4-DOF (3 revolute and 1 prismatic joints) robot model that incorporates disturbances due to sea waves as illustrated in Fig. 2. This would correspond to assuming that the disturbance influence on the robot can be described with only these joints and remains the same for a travelling and a stationary vessel.

Since we are not interested in the motion of the vehicle but in the motion of the robot, the effect of the disturbance should be transformed onto the robot arm. Thus, following [15] and premultiplying the transformation matrix from base to the tip of the disturbance model with the homogeneous transformation matrix of the robot arm and using the rules in [7], the dynamics equation of the whole system is obtained. As expected, the resulting equation for the robot arm contains highly nonlinear functions of $\alpha, \beta, \gamma, d, \theta, \psi$, and their derivatives.

Several works on the modeling of sea waves, like [17], [12], and [15], focus on finding energy spectrum of the waves and propose a rich class of disturbance signals. Singling out 


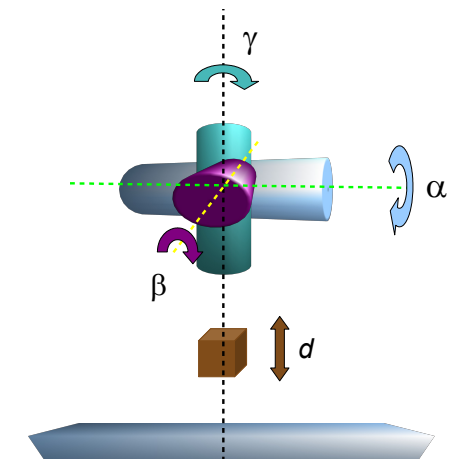

Fig. 2. The Base Disturbance Model

elementary signals step, ramp, sinusoidal signals, and their combinations and assuming small amplitudes, the disturbance on the robot is modeled here as entering (2) as

$\mathbf{f}_{\mathbf{i}}=\frac{\tau_{i}-N_{i}-G_{i}-\tau_{f i}-\tau_{\text {dist }_{i}}}{M_{i i}} ; i=1,2$.

We will thus work with this simplified sea wave disturbance model in synthesizing controllers. This assumption corresponds to reflecting the base disturbance as step, ramp, or sinusoidal torques acting directly on joints.

\section{LINEAR CONTROLLER}

\section{A. Linearization of the Model}

Linearization of (2) without considering friction torques $\left(\tau_{\mathbf{f}}=[0,0]^{T}\right)$ about the equilibrium values

$$
\theta^{*}, \dot{\theta}^{*}=0, \psi^{*}, \dot{\psi}^{*}=0, \tau_{1}^{*}=0, \tau_{2}^{*}(t)=-\kappa \cos \left(\psi^{*}+\phi\right),
$$

with arbitrary but fixed constants for $\theta^{*}, \psi^{*}$, gives the statespace equations

$\frac{\mathbf{d}}{\mathbf{d t}}\left[\begin{array}{c}\theta \\ \dot{\theta} \\ \psi \\ \dot{\psi}\end{array}\right]=\mathbf{A}\left[\begin{array}{c}\theta-\theta^{*} \\ \dot{\theta} \\ \psi-\psi^{*} \\ \dot{\psi}\end{array}\right]+\mathbf{B}\left[\begin{array}{c}\tau_{1} \\ \tau_{2}-\tau_{2}^{*}\end{array}\right], \mathbf{y}=\left[\begin{array}{c}\theta \\ \dot{\theta} \\ \psi \\ \dot{\psi}\end{array}\right]$

$$
\begin{aligned}
\mathbf{A} & =\left[\begin{array}{cccc}
0 & 1 & 0 & 0 \\
0 & 0 & 0 & 0 \\
0 & 0 & 0 & 1 \\
0 & 0 & \frac{-\kappa \sin \left(\psi^{*}+\phi\right)}{\eta} & 0
\end{array}\right], \\
\mathbf{B} & =\left[\begin{array}{cc}
0 & 0 \\
\frac{1}{\zeta+\gamma \cos ^{2}\left(\psi^{*}\right)-p \sin \left(2 \psi^{*}\right)} & 0 \\
0 & 0 \\
0 & \frac{1}{\eta}
\end{array}\right] .
\end{aligned}
$$

Note that, linearization with respect to $\mathrm{x}$ eliminates the terms that arise from coriolis and centrifugal forces but retains the unbalance term in matrix $\mathbf{A}$ in a different form. We compensate for this nonlinear term as part of the overall controller and then design a linear controller for the resulting linear plant $(\widehat{P})$. Here, by unbalance compensation we mean gravity compensation as in Fig. 3.

\section{B. Required Internal Model}

In Fig. 3, $P$ is the original nonlinear plant represented by (2) that will be compared with the linearized plant $\widehat{P}$, combination of the plant (4) and the compensation for unbalance. It can be shown that $\widehat{P}$ has the transfer matrix

$\widehat{P}(s)=\left[\begin{array}{cc}\frac{p_{1}(s)}{q_{1}(s)} & 0 \\ 0 & \frac{p_{2}(s)}{q_{2}(s)}\end{array}\right]=\left[\begin{array}{cc}\frac{1}{J_{1} s^{2}} & 0 \\ 0 & \frac{1}{J_{2} s^{2}}\end{array}\right]$

where the inertias $J_{i}, i=1,2$, are constant around the equilibrium point. The two channels of the plant are noninteracting so that for diagonal reference and disturbance inputs $R, D$ in Fig. 3, we will synthesize a diagonal controller $C$.

In Fig. 3, if the linearized plant $\widehat{P}$ is replaced by $P$, then the two channels are decoupled and controllers $\frac{p_{c}}{q_{c}}$ in the two channels can be synthesized independently to achieve internal stability, tracking, and disturbance regulation simultaneously. Focusing on a single channel, we need to choose polynomials $p_{c}, q_{c}$ of $s$ such that the characteristic polynomial $\Delta(s)=p p_{c}+q q_{c}$ is Hurwitz (all roots in the strict left half complex plane $\mathbf{C}_{-}$) and reference-to-output, disturbance-tooutput transfer functions are stable (all poles in $\mathbf{C}_{-}$). If we write, for anti-Hurwitz (all roots in the closed right half plane) polynomials $r(s), d(s)$,

$R(s)=\frac{1}{r(s)}, \quad D(s)=\frac{1}{d(s)}$,

then these requirements are that

- $\Delta(s)$ is a Hurwitz polynomial (internal stability),

- $\frac{q(s) q_{c}(s)}{r(s) \Delta(s)}$ is stable (tracking),

- $\frac{p(s) q_{c}(s)}{d(s) \Delta(s)}$ is stable (regulation).

An additional technical requirement that should be taken into account in synthesizing $p_{c}(s), q_{c}(s)$ is that $C(s)$ is proper (realizable). Note that if sinusoidal and ramp signals are to be tracked and/or regulated, then in frequency domain one can choose $r(s)=s^{2}\left(s^{2}+w_{0}^{2}\right)$ and $d(s)=s^{2}\left(s^{2}+w_{1}^{2}\right)$ for frequencies $w_{0}, w_{1}$.

Let $G C D(r, q)$ denote the monic greatest common divisor of the two polynomials $r$ and $q$ and let $\operatorname{LCM}(r, q)$ denote

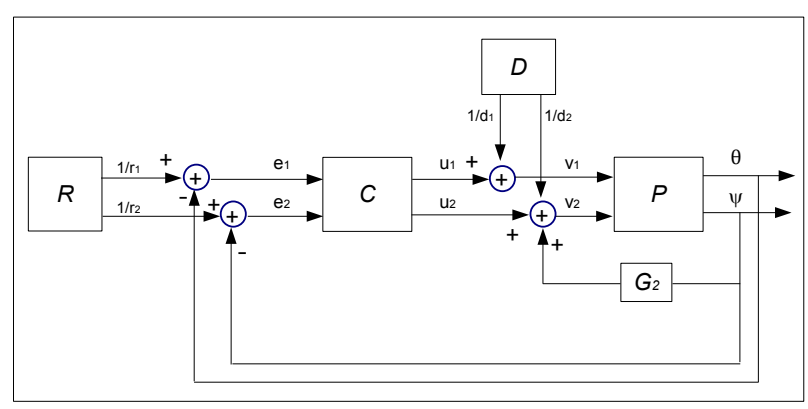

Fig. 3. System with Linear Controller 
their monic least common multiple. Then, the internal model principle for this combined tracking and regulation problem is that

$l:=\operatorname{LCM}\left(\frac{d}{G C D(d, p)}, \frac{r}{G C D(r, q)}\right)$ divides $q_{c}$,

which states that the poles (with multiplicities) of the disturbance generating system $D$ that are not among plant zeros and of the poles of the reference generating system $R$ that are not among plant poles combined must be included among the controller poles. Then, a necessary and sufficient condition for being able to synthesize a $C$ that achieves the three objectives simultaneously is that $(l, p)$ is coprime, [20].

We now examine the stability and performance of controllers synthesized via the principle (5) for basic step, ramp, and sinusoidal reference and/or disturbance signals. The frequency of the sinusoids are assumed to be known exactly but the amplitudes of steps and sinusoids and slopes of ramps need not be known. It follows by (5) that, for the double integrator plant under consideration, $P D$ and $P I D$ controllers would be able to track step and ramp references simultaneously and PID would be able to regulate step disturbances; but, both would not be able to track or regulate any sinusoidal signal. Neither would they be able to regulate ramp disturbances since the single pole at the origin of such controllers would not satisfy (5).

For instance, if a $P I D$ controller is used for $C$, then in terms of constants $K_{d}, K_{p}, K_{i}$, we have

$$
\begin{aligned}
& C(s)=\frac{p_{c}}{q_{c}}=\frac{K_{d} s^{2}+K_{p} s+K_{i}}{s} ; \\
& H(s)=\frac{K_{d} s^{2}+K_{p} s+K_{i}}{\Delta(s)} ; \\
& \Delta(s)=J s^{3}+K_{d} s^{2}+K_{p} s+K_{i} .
\end{aligned}
$$

where $H$ is the closed loop transfer function. By RouthHurwitz criterion, the polynomial $\Delta(s)$ is Hurwitz just in case $K_{i}>0$ and $J K_{i}<K_{p} K_{d}$. The controller with such choices would track step and ramp trajectories, but will not be able to track sinusoidal references. Also, it would regulate step disturbances but not ramp and sinusoidal disturbances. As an example, Fig. 4 illustrates disturbance regulation performance of $P I D$ controller.

\section{A Linear Higher-Order Controller}

$P D$ and $P I D$ type controllers are not able to regulate sinusoidal disturbances. Assume that the frequency of the sinusoidal torque disturbance at the joints which comes from sea waves is known and it is $w_{0}$. Then, by (5), the controller must be in the form

$C(s)=\frac{p_{c}}{q_{c}}=\frac{p_{c}}{s^{2}\left(s^{2}+w_{0}^{2}\right) \overline{q_{c}}}$

for some polynomial $\overline{q_{c}}$ to be determined, together with $p_{c}$ to satisfy the internal stability requirement. For this purpose, let us seek a minimal degree controller

$C(s)=\frac{p_{c}}{q_{c}}=\frac{a s^{5}+b s^{4}+c s^{3}+d s^{2}+e s+f}{s^{2}\left(s^{2}+w_{0}^{2}\right)(s+\mu)}$,
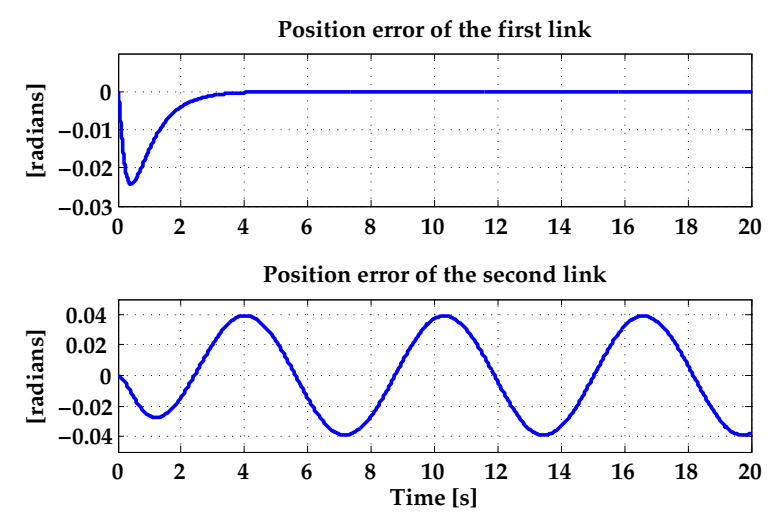

Fig. 4. System response to zero position command under step (for the first link) and sinusoidal (for the second link) torque disturbances with $P I D$ Controller

for some seven parameters $a, b, c, d, e, f, \mu$. The characteristic polynomial is

$$
\begin{aligned}
\Delta(s)= & J s^{7}+J \mu s^{6}+\left(J w_{0}^{2}+a\right) s^{5}+\left(J w_{0}^{2} \mu+b\right) s^{4} \\
& +c s^{3}+d s^{2}+e s+f .
\end{aligned}
$$

For the purpose of simulation, we place all zeros of $\Delta$ at $s=-1$ through the choice

$$
\begin{aligned}
& \Delta(s)=(s+1)^{7}, \quad a=J\left(21-w_{0}^{2}\right), \quad b=J\left(35-w_{0}^{2} \mu\right), \\
& c=35 J, \quad d=21 J, \quad e=7 J, \quad f=J, \quad \mu=7 .
\end{aligned}
$$

The constants $J_{i} ; i=1,2$ around the equilibrium point $\theta^{*}=$ $0 ; \psi^{*}=0$ are found from the state-space equation of the linearized system (4).

In figures 4-6, some of the simulation results are displayed for zero reference inputs. In Fig. 4, PID controller, while in Fig. 5 and 6, the proposed controller is applied. In Fig. 4, the sinusoidal disturbance in the second link is not regulated. In Fig. 5, the position errors go to zero, which means that the system regulates step and ramp disturbances. In Fig. 6, for the first link $(\theta)$, the frequency of the disturbance signal is $w_{0}$; so, as expected, the sinusoidal disturbance is regulated. While in the other link $(\psi)$, because the sinusoidal disturbance applied has a different frequency, the proposed controller fails. Note that the settling time in these figures can be arbitrarily shortened by a better choice of the characteristic roots.

Table 1 compares the performances of controllers used. It is seen that proposed controllers are able not only to track step, ramp, and sinusoidal reference signals, but also to regulate the torque disturbances of the same form while PID controllers have deficiency both in tracking and regulation problems.

\section{CONCLUSIONS}

Computed Torque Control algorithms require perfect knowledge of the system nonlinearities and the signal to be tracked in order to achieve internal stability and tracking. Internal model principle can not be directly applied to these controllers 

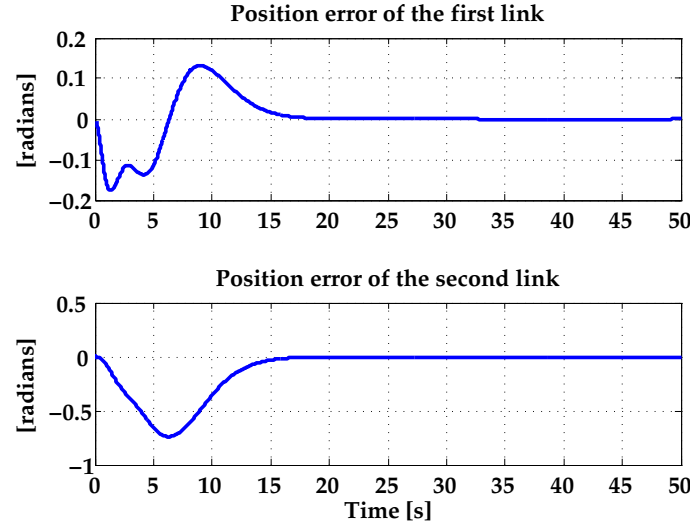

Fig. 5. System response to zero position command under step (for the first link) and ramp (for the second link) disturbances with the Proposed Controller
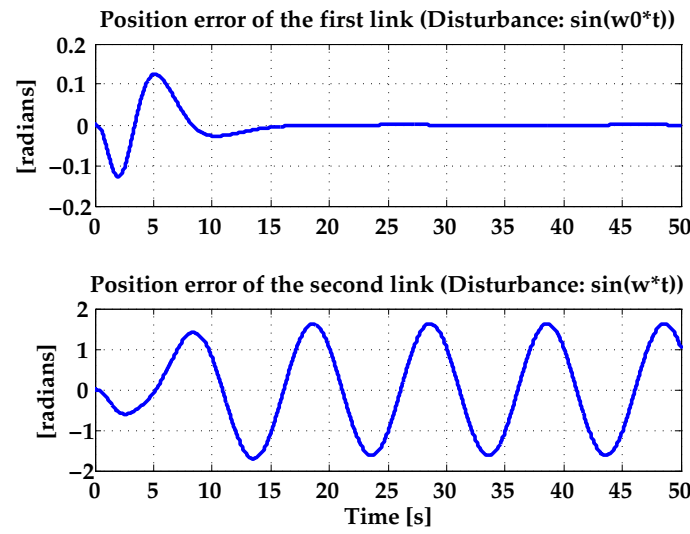

Fig. 6. System response to zero position command under sinusoidal disturbances with different frequencies with the Proposed Controller

TABLE I

PERFormances of CONTROLleRs USED

\begin{tabular}{|c|c|c|}
\hline \multirow{2}{*}{ Reference } & \multicolumn{2}{|c|}{ Controller } \\
& PID & Proposed \\
\hline Step & Yes & Yes \\
\hline Ramp & Yes & Yes \\
\hline Sin $\left(w_{0} t\right)$ & No & Yes \\
\hline \hline \multirow{2}{*}{ Disturbance } & \multicolumn{2}{|c|}{ Controller } \\
& PID & Proposed \\
\hline Step & Yes & Yes \\
\hline Ramp & No & Yes \\
\hline Sin $\left(w_{0} t\right)$ & No & Yes \\
\hline
\end{tabular}

for the purpose of disturbance regulation; and estimationbased structures oriented towards regulation may be infeasible for some plants (e.g., systems with uncertainties, [13]). We hence search for a linear controller synthesized by evoking the internal model principle, which will be directly applied to the original nonlinear plant. We show with simulations that using only internal model principle and treating the sea disturbances as external disturbances, we can synthesize a linear controller that solves tracking and regulation problems and keeps the system internally stable as well. It should be noted that, our assumption that the effects of sea disturbances on the robot arm can be modeled as an external torque disturbance acting at the joints helps the synthesis of a servomechanism but may be rather severe in some applications. PID controllers also perform as would be expected by the principle of internal model and they are able to track and regulate step signals. Their ability to track ramp commands and failure to regulate ramp disturbances is an indication of the fact that the nonlinear plant behaves like it has a pole at the origin.

A cautionary remark on linearization is that the unbalance term, if included in linearization, restricts the operating range in which a linear controller can perform. It is thus better to compensate the unbalance term, when it exists, at the outset with a nonlinear feedback and synthesize the internal model controller subsequently.

Our results establish that the nonlinear dynamic model of the robot (1) behaves like its linearized version (4) once the unbalance term is compensated; and that, a linear controller performs well with respect to tracking and disturbance regulation objectives.

\section{ACKNOWLEDGEMENT}

This work was supported by ASELSAN Inc., Contract No: SST-1A-2011-034.

\section{REFERENCES}

[1] Andrews B. W., E. D. Sontag, and P. A. Iglesias, "An approximate internal model principle: Applications to nonlinear models of biological systems, " in Proc. 17th IFAC World Congress, Seoul, pp. 15873-15878, 2008.

[2] Bejczy A. K. , T. J. Tarn, X. Yun, and S. Han, "Nonlinear feedback control of PUMA 560 robot arm by computer," 24th IEEE Conference on Decision and Control, vol.24, pp.1680-1688, Dec. 1985.

[3] Byrnes C. I., F. Delli Priscoli, and A. Isidori, Output regulation of uncertain nonlinear systems. Boston: Birkhauser, 1997.

[4] Francis B. A. and W. M. Wonham, "The internal model principle for linear multivariable regulators, " J. Appl. Math. Optimiz. vol 2, pp.170194, 1975

[5] From P. J., V. Duindam, J. T. Gravdahl, and S. Sastry, "Modeling and motion planning for mechanisms on a non-inertial base," International Conference on Robotics and Automation, Kobe, Japan, 2009, pp.33203326.

[6] From P. J., J. T. Gravdahl, and P. Abbeel, "On the influence of ship motion prediction accuracy on motion planning and control of robotic manipulators on seaborne platforms, " 2010 IEEE International Conference on Robotics and Automation (ICRA), pp.5281-5288, 2010.

[7] Fu K. S., R. C. Gonzalez, and C. S. G. Lee, Robotics: Control, Sensing, Vision, and Intelligence. New York: McGraw-Hill, 1987.

[8] Hepburn J. S. A. and W. M. Wonham, "Error feedback and internal models on differentiable manifolds, "IEEE Trans. Automatic Control vol. 29 , pp.397-403, 1984

[9] Hepburn J. S. A. and W. M. Wonham, "The semistable-center-unstable manifold near a critical element, " J. Math. Anal. Appl., vol. 103, pp.321331,1984

[10] Hepburn J. S. A. and W. M. Wonham, "Structurally stable nonlinear regulation with step inputs, "Mathematical Systems Theory, vol. 17, pp.319-333, 1984.

[11] Jayawardhana B. and G. Weiss, "Tracking and disturbance rejection for fully actuated mechanical systems", Automatica, Volume 44, Issue 11, November 2008, pp.2863-2868.

[12] Lantos B. and Marton L., Nonlinear Control of Vehicles and Robots, Springer Verlag, London, 2011. 
[13] Lewis F. L., D. M. Dawson, and C. T. Abdallah, Robot manipulator control: theory and practice. Marcel Dekker, Inc., 2004.

[14] Li Q., A. N. Poo, and C. M. Lim, "Internal model structure in the control of robot manipulators," Mechatronics, Volume 6, Issue 5, Aug. 1996, pp.571-590.

[15] Love L. J., J. F. Jansen, and F. G. Pin, "On the modeling of robots operating on ships, " IEEE International Conference on Robotics and Automation, pp. 2436-2443, 2004

[16] Neuman C. P. and J. J. Murray, "The Complete Dynamic Model and Customized Algorithms of the Puma Robot, "IEEE Transactions on Systems, Man and Cybernetics, vol.17, no.4, pp.635-644, July 1987.

[17] Perez T., Ship motion control, course keeping and roll stabilization using rudder and fins. London, Springer-Verlag, 2005.

[18] Ramasubramanian A. and L. R. Ray, "Adaptive friction compensation using extended Kalman-Bucy filter friction estimation: A comparative study," in Proc. Amer. Contr. Conf., Jun. 2000, pp.2588-2594.

[19] Spong M. W., S. Hutchinson, and M. Vidyasagar, Robot modeling and control. John Wiley \& Sons, Inc., New York, 2006.

[20] Website. http://www.ee.bilkent.edu.tr/\%7Eguler/GO2012PaperAppendix 